\title{
Reliability Parameter Interval Estimation of NC Machine Tools considering Working Conditions
}

\author{
Li Hongzhou ${ }^{1,2}$ and Sun Lixia ${ }^{1,3}$ \\ ${ }^{1}$ College of Mechanical Engineering, Beihua University, Jilin 132013, China \\ ${ }^{2}$ College of Mechanical Science and Engineering, Jilin University, Changchun 130025, China \\ ${ }^{3}$ College of Mechanical and Electric Engineering, Changchun University of Science and Technology, Changchun 130025, China
}

Correspondence should be addressed to Sun Lixia; sunlixiajilin@sohu.com

Received 4 March 2017; Accepted 17 August 2017; Published 26 September 2017

Academic Editor: Alessandro Gasparetto

Copyright (C) 2017 Li Hongzhou and Sun Lixia. This is an open access article distributed under the Creative Commons Attribution License, which permits unrestricted use, distribution, and reproduction in any medium, provided the original work is properly cited.

\begin{abstract}
Aiming at the problem that the parameter interval estimation of NC machine tool's reliability model considering working conditions established by Hongzhou is difficult to implement, given that it has several independent variables, an improved interval estimation method based on Bootstrap is proposed. Firstly, the two-step estimation method was used to calculate the point estimation of NC machine tool's reliability parameter in test field, based on which $B$ resamplings are generated based on the point estimation. The reliability parameter's point estimation of the resamplings was obtained by maximum likelihood estimation. Permutation of $B$ point estimations was made in ascending order and the interval estimations were obtained by the $\alpha$ quantile of the permutation. Case study indicated that the location and length of the interval estimation of NC machine tools' reliability parameter, under different levels of working condition covariates, vary obviously.
\end{abstract}

\section{Introduction}

The reliability model of NC machine tools considering working conditions was established in [1], and the point estimations of the shape parameter, scale parameter, and the coefficients of working condition covariates were obtained by the two-step estimation method. To make NC machine tool's reliability evaluation more accurate, the interval estimations of the shape parameter, scale parameter, and the coefficients of working condition covariates should be made. The commonly used interval estimation methods include Fisher information matrix method [2], likelihood ratio interval estimation [3, 4], pivot method [5], and maximum likelihood interval estimation [6]. Each of the above interval estimation methods usually establishes, respectively, the interval estimation formula with only one independent variable. However, there are several independent variables of the reliability model in [1], including Time between Failure (TBF) and working condition covariates, which make it difficult for the above methods to calculate the model parameters' interval estimation.

The Bootstrap method [7] only depends on a lot of resamples to calculate interval estimation overcoming the shortcomings of the other interval estimation methods which need to construct the complex formula.

Aiming at the above problem, a reliability model parameter's interval estimation method of NC machine tools considering working conditions based on the Bootstrap method is proposed in this paper. Firstly, parameter's point estimation based on the NC machine tools' test sample is obtained by two-step estimation method; the resampling is obtained by Bootstrap based on the parameter point estimations of the NC machine tools' test sample. The parameter point estimations of each resampling are calculated by the maximum likelihood estimation. The parameter point estimations under every working condition level are obtained by conversion equation. Finally, the feasibility of the proposed method is validated in the case study. 


\section{Reliability Model of NC Machine Tools considering Working Conditions}

For convenience, the failure rate function of NC machine tools considering working conditions in [1] is expressed as follows:

$$
\lambda\left(\frac{t}{X}\right)=\frac{m}{\eta}\left(\frac{t}{\eta}\right)^{m-1} \cdot \exp \left[\beta\left(X-X_{1}\right)\right]
$$

where $t$ is the Time between Failures (a random variable) of NC machine tools; $X=\left(X_{1}, X_{2}, \ldots, X_{i}, \ldots, X_{n}\right), X$ is the vector of working condition covariates, which affects the failure rate of NC machine tools, and $X_{i}$ is the $i$ th covariate, such as cutting force, environment temperature, and number of tool changes or vibration; $m$ is the shape parameter under $X_{1}$, and $m>0 ; \eta$ is the scale parameter under $X_{1}, \eta>0$; $\beta=\left(\beta_{1}, \beta_{2}, \ldots, \beta_{i}, \ldots, \beta_{n}\right)$ is the vector of $X$ 's coefficients, which reflect the covariates' influences on the failure rate function, and $\beta_{i}$ is the coefficient of $X_{i}$.

So, the reliability function of NC machine tools considering working conditions is expressed as follows:

$$
R(t, \mathbf{X})=\left\{\exp \left[-\left(\frac{t}{\eta}\right)^{m}\right]\right\}^{\exp \left[\boldsymbol{\beta}\left(\mathbf{X}-\mathbf{X}_{1}\right)\right]}
$$

Suppose that the failure rate function of two-parameter Weibull distribution of NC machine tools under covariate $X_{i}$ is

$$
\lambda\left(\frac{t}{X_{i}}\right)=\frac{m}{\eta_{i}}\left(\frac{t}{\eta_{i}}\right)^{m-1}
$$

where $m$ is the shape parameter; $\eta_{i}$ is the scale parameter under covariate $X_{i}$.

Substituting (3) in (1) gets the scale parameter $\eta_{i}$ :

$$
\eta_{i}=\frac{\eta}{\exp \left[(\beta / m)\left(X_{i}-X_{1}\right)\right]} .
$$

\section{Reliability Parameter Interval Estimations of NC Machine Tools considering Working Conditions}

3.1. Bootstrap Method. Bootstrap method generates new samples by drawing samples from the original samples, obtaining the so-called resamplings, which can be used for parameter's interval estimation. The basic idea of the Bootstrap resampling is as follows $[8,9]$.

Suppose $X=\left(x_{1}, x_{2}, \ldots, x_{k}\right)$ is a sample from population $F(x \mid \theta)$ with parameter $\theta$ which is equivalent to the maximum likelihood estimation $\widehat{\theta}$.

Based on $F(x \mid \theta), B$ Bootstrap resamplings $\left\{X^{1}, X^{2}, \ldots\right.$, $\left.X^{b}, \ldots, X^{B}\right\}$ are drawn, where the size of each resampling is $l$, and $X^{b}=\left(x_{1}^{b}, x_{2}^{b}, \ldots, x_{l}^{b}\right)$ is the $b$ th Bootstrap resampling. Based on each Bootstrap resampling, the parameters' estimations are calculated to be $\left\{\widehat{\theta}^{1}, \widehat{\theta}^{2}, \ldots, \widehat{\theta}^{B}\right\}$.
Arranging $\left\{\widehat{\theta}^{1}, \widehat{\theta}^{2}, \ldots, \widehat{\theta}^{B}\right\}$ in ascending order obtains $\left\{\widehat{\theta}^{* 1}, \widehat{\theta}^{* 2}, \ldots, \widehat{\theta}^{* B}\right\}$, and the interval estimation of parameter at the confidence level $\alpha$ is as follows:

$$
\left[\widehat{\theta}_{\mathrm{lo}}, \widehat{\theta}_{\mathrm{up}}\right]=\left[\widehat{\theta}^{*(\alpha / 2 \times B)}, \widehat{\theta}^{*((1-\alpha / 2) \times B)}\right]
$$

where $\widehat{\theta}_{\text {lo }}$ is the lower limit of the interval estimation; $\widehat{\theta}_{\text {up }}$ is the upper limit of the interval estimation.

\subsection{Reliability Parameter Interval Estimation's Step of NC Machine Tools considering Working Conditions}

Step 1. Point estimations, including $\widehat{m}, \widehat{\eta}$, and $\widehat{\beta}$, of the shape parameter $m$, the scale parameter $\eta$, and coefficients of working condition covariates $\beta$ are calculated by two-step interval estimation in [1], according to the fault information and the working conditions corresponding to fault information obtained from the test field.

Step 2. B Bootstrap resamplings with the fault information and the working conditions are obtained by sampling based on the point estimations $\widehat{m}, \widehat{\eta}$, and $\widehat{\beta}$ in Step 1 .

Step 3. The maximum likelihood estimation method is adopted to estimate parameters $\beta, m$, and $\eta$ estimation in (1) for each resampling, then point estimations $\widehat{m}_{b}, \widehat{\eta}_{b}$, and $\widehat{\beta}_{b}$ are obtained, and the subscript $b$ is the $b$ th resampling, $b=1,2, \ldots, B$.

The likelihood function is given as

$$
\begin{aligned}
L(\eta, m) & =\prod_{i=1}^{r} f\left(t_{i}, \mathbf{X}_{i}\right) \prod_{i=r+1}^{n} R\left(t_{i}, \mathbf{X}_{i}\right) \\
& =\prod_{i=1}^{r}\left\{\left\{\exp \left[-\left(\frac{t_{i}}{\eta}\right)^{m}\right]\right\}^{\exp \left[\boldsymbol{\beta}\left(\mathbf{X}_{i}-\mathbf{X}_{1}\right)\right]} \cdot \frac{m}{\eta}\left(\frac{t_{i}}{\eta}\right)^{m-1}\right. \\
\cdot & \left.\exp \left[\boldsymbol{\beta}\left(\mathbf{X}_{i}-\mathbf{X}_{1}\right)\right]\right\} \\
& \cdot \prod_{i=r+1}^{n}\left\{\exp \left[-\left(\frac{t_{i}}{\eta}\right)^{m}\right]\right\}^{\exp \left[\boldsymbol{\beta}\left(\mathbf{X}_{i}-\mathbf{X}_{1}\right)\right]}
\end{aligned}
$$

Take the logarithm of both sides in (6); then

$$
\begin{aligned}
\operatorname{Ln}[L(\eta, m)]= & r \ln m-r m \ln \eta \\
& +(m-1) \ln \left(t_{1} t_{2} \ldots t_{r}\right) \\
& -\left[\left(\frac{t_{1}}{\eta}\right)^{m}+\left(\frac{t_{2}}{\eta}\right)^{m}+\cdots+\left(\frac{t_{r}}{\eta}\right)^{m}\right] \\
& +\boldsymbol{\beta}\left(\mathbf{X}_{1}+\mathbf{X}_{2}+\cdots+\mathbf{X}_{r}-r \mathbf{X}_{1}\right) \\
& +\sum_{i=r+1}^{n}\left[-\left(\frac{t_{i}}{\eta}\right)^{m} \exp \left[\boldsymbol{\beta}\left(\mathbf{X}_{i}-\mathbf{X}_{1}\right)\right]\right]
\end{aligned}
$$


Take the partial derivatives of the parameters $\beta, \eta$, and $m$ in (7), respectively, and then

$$
\begin{aligned}
& \frac{\partial L n[L(\eta, m)]}{\partial \beta_{1}}=(1-r) \mathbf{X}_{1} \\
& +\sum_{i=r+1}^{n}\left[-\left(\frac{t_{i}}{\eta}\right)^{m}(1-r) \mathbf{X}_{1} \cdot \exp \left[\boldsymbol{\beta}\left(\mathbf{X}_{i}-\mathbf{X}_{1}\right)\right]\right] \\
& \frac{\partial \operatorname{Ln}[L(\eta, m)]}{\partial \beta_{i}}=\beta \mathbf{X}_{i} \\
& +\sum_{i=r+1}^{n}\left[-\left(\frac{t_{i}}{\eta}\right)^{m} \mathbf{X}_{i} \exp \left[\boldsymbol{\beta}\left(\mathbf{X}_{i}-\mathbf{X}_{1}\right)\right]\right] \\
& i=2,3, \ldots, n \\
& \frac{\partial L n[L(\eta, m)]}{\partial \eta}=-\frac{r m}{\eta}+\left[m t_{1}^{m} \eta^{(-m-1)}+m t_{2}^{m} \eta^{(-m-1)}\right. \\
& \left.+\cdots+m t_{r}^{m} \eta^{(-m-1)}\right] \\
& +\sum_{i=r+1}^{n}\left[m t_{i}^{m} \eta^{(-m-1)} \exp \left[\boldsymbol{\beta}\left(\mathbf{X}_{i}-\mathbf{X}_{1}\right)\right]\right] \\
& \frac{\partial L n[L(\eta, m)]}{\partial m}=\frac{r}{m}-r \ln \eta+\ln \left(t_{1} t_{2} \cdots t_{r}\right) \\
& -\left[\left(\frac{t_{1}}{\eta}\right)^{m} \ln \frac{t_{1}}{\eta}+\left(\frac{t_{2}}{\eta}\right)^{m} \ln \frac{t_{2}}{\eta}+\cdots\right. \\
& \left.+\left(\frac{t_{r}}{\eta}\right)^{m} \ln \frac{t_{r}}{\eta}\right] \\
& +\sum_{i=r+1}^{n}\left[-\left(\frac{t_{i}}{\eta}\right)^{m} \ln \left(\frac{t_{i}}{\eta}\right) \exp \left[\boldsymbol{\beta}\left(\mathbf{X}_{i}-\mathbf{X}_{1}\right)\right]\right] .
\end{aligned}
$$

Since (8) have no analytical solutions, Newton-Raphson [10] numerical algorithm is used to estimate parameters $\beta, \eta$, and $m$.

Step 4. The scale parameter $\eta_{b i}$ of two-parameter Weibull distribution of NC machine tools under covariate $X_{i}$ in the $b$ th resampling is obtained based on (4).

$$
\begin{aligned}
\eta_{b i}=\frac{\eta_{b}}{\exp \left[\left(\beta_{b} / m_{b}\right)\left(X_{i}-X_{1}\right)\right]} \\
\quad b=1,2, \ldots, B, i=1,2, \ldots, u,
\end{aligned}
$$

where $u$ represents number of covariates' levels.

Step 5. According to the scale parameter $\eta_{b i}$ obtained by Step 4 and shape parameter $m$ obtained by Step 3, the MTBF of NC machine tools under covariate $X_{i}$ in the $b$ th resampling is

$$
\begin{aligned}
\operatorname{MTBF}_{b i}=\widehat{\eta}_{b i} * \Gamma\left(1+\frac{1}{\widehat{m}_{b}}\right) \\
\quad b=1,2, \ldots, B, i=1,2, \ldots, u .
\end{aligned}
$$

Step 6 . The shape parameters $\widehat{m}_{1}, \ldots, \widehat{m}_{B}$ obtained by Step 3 are arranged in ascending order and then get sequence

$$
\widehat{m}^{* 1}, \ldots, \widehat{m}^{* B} \text {. }
$$

Step 7 . The scale parameters $\hat{\eta}_{b i}, i=1,2, \ldots, u$ obtained by Steps 3 and 4 are arranged, respectively, in ascending order and then get sequence

$$
\begin{gathered}
\left(\widehat{\eta}^{* 11}, \ldots, \widehat{\eta}^{* 1 b}, \ldots, \widehat{\eta}^{* 1 B}\right) \\
\left(\widehat{\eta}^{* 21}, \ldots, \widehat{\eta}^{* 2 b}, \ldots, \widehat{\eta}^{* 2 B}\right) \\
\vdots \\
\left(\widehat{\eta}^{* u 1}, \ldots, \widehat{\eta}^{* u b}, \ldots, \widehat{\eta}^{* u B}\right) .
\end{gathered}
$$

Step 8 . The coefficients $\widehat{\beta}_{1}, \ldots, \widehat{\beta}_{k}$ of working condition covariates obtained by Step 3 are arranged in ascending order and then get sequence

$$
\left(\widehat{\beta}^{* i 1}, \ldots, \beta^{* i B}\right), \quad i=1,2, \ldots, k .
$$

Step 9. $\mathrm{MTBF}_{b i}, i=1,2, \ldots, u$ obtained by Step 5 are arranged in ascending order and then get sequence

$$
\left(\mathrm{MTBF}^{* i 1}, \ldots, \mathrm{MTBF}^{* i B}\right) \quad i=1,2, \ldots, u .
$$

Step 10. According to Step 6, set up confidence level $1-\alpha$ and solve and round $B \cdot(\alpha / 2)$ and $B \cdot(1-\alpha / 2)$, respectively; then the interval estimations of the shape parameter are obtained as follows:

$$
\left[\widehat{m}^{* B \cdot(\alpha / 2)}, \widehat{m}^{* B \cdot(1-\alpha / 2)}\right]
$$

where $\widehat{m}^{* B \cdot(\alpha / 2)}$ is the lower limit of interval estimation of the shape parameter at the confidence level $1-\alpha \cdot \widehat{m}^{* B \cdot(1-\alpha / 2)}$ is the upper limit of interval estimation of the shape parameter at the confidence level $1-\alpha$.

Step 11. According to Step 7, set up confidence level $1-\alpha$ and solve and round $B \cdot(\alpha / 2)$ and $B \cdot(1-\alpha / 2)$, respectively; then the interval estimations of the scale parameter under the $i$ th covariate level are obtained:

$$
\left[\widehat{\eta}^{* i, B \cdot(\alpha / 2)}, \widehat{\eta}^{* i, B \cdot(1-\alpha / 2)}\right] \quad i=1,2, \ldots, \mathcal{u},
$$

where $\widehat{\eta}^{* i, B \cdot(\alpha / 2)}$ is the lower limit of interval estimation of the scale parameter under the $i$ th covariate level at the confidence level $1-\alpha . \hat{\eta}^{* i, B \cdot(1-\alpha / 2)}$ is the upper limit of interval estimation of the shape parameter under the $i$ th covariate level at the confidence level $1-\alpha$.

Step 12. According to Step 8, set up confidence level $1-\alpha$ and solve and round $B \cdot(\alpha / 2)$ and $B \cdot(1-\alpha / 2)$, respectively; then the interval estimations of the coefficients of the $i$ th working condition covariate are obtained:

$$
\left[\widehat{\beta}^{* i, B \cdot(\alpha / 2)}, \widehat{\beta}^{* i, B \cdot(1-\alpha / 2)}\right] \quad i=1,2, \ldots, k,
$$


TABLE 1: Failure data of NC machine tools under different working conditions covariates.

\begin{tabular}{|c|c|c|c|c|c|c|}
\hline Workpiece Name & Cutting force/KN & $\begin{array}{l}\text { Number of tool } \\
\text { changes } /(N / \mathrm{h})\end{array}$ & Cutting Fluid & Temperature $/{ }^{\circ} \mathrm{C}$ & $\mathrm{TBF} / \mathrm{h}$ & Data type \\
\hline Flywheel & 0.35 & 2 & 1 & 20 & 437 & 1 \\
\hline Flywheel & 0.35 & 2 & 1 & 20 & 1896 & 1 \\
\hline Flywheel & 0.35 & 2 & 1 & 20 & 340 & 1 \\
\hline Flywheel & 0.35 & 2 & 1 & 20 & 244 & 1 \\
\hline Flywheel & 0.35 & 2 & 1 & 20 & 249 & 1 \\
\hline Flywheel & 0.35 & 2 & 1 & 20 & 898 & 1 \\
\hline Flywheel & 0.35 & 2 & 1 & 20 & 1148 & 0 \\
\hline Cylinder Block & 0.43 & 17 & 0 & 21 & 158 & 1 \\
\hline Cylinder Block & 0.43 & 17 & 0 & 21 & 67 & 1 \\
\hline Cylinder Block & 0.43 & 17 & 0 & 21 & 242 & 1 \\
\hline Cylinder Block & 0.43 & 17 & 0 & 21 & 107 & 1 \\
\hline Cylinder Block & 0.43 & 17 & 0 & 21 & 155 & 1 \\
\hline Cylinder Block & 0.43 & 17 & 0 & 21 & 1717 & 1 \\
\hline Cylinder Block & 0.43 & 17 & 0 & 21 & 812 & 1 \\
\hline Cylinder Block & 0.43 & 17 & 0 & 21 & 724 & 1 \\
\hline Cylinder Head & 0.54 & 4 & 0 & 22 & 316 & 1 \\
\hline Cylinder Head & 0.54 & 4 & 0 & 22 & 99 & 1 \\
\hline Cylinder Head & 0.54 & 4 & 0 & 22 & 1419 & 1 \\
\hline Cylinder Head & 0.54 & 4 & 0 & 22 & 1430 & 1 \\
\hline Cylinder Head & 0.54 & 4 & 0 & 22 & 225 & 1 \\
\hline Cylinder Head & 0.54 & 4 & 0 & 22 & 773 & 1 \\
\hline Cylinder Head & 0.54 & 4 & 0 & 22 & 843 & 0 \\
\hline Mould & 0.78 & 10 & 1 & 19 & 398 & 1 \\
\hline Mould & 0.78 & 10 & 1 & 19 & 29 & 1 \\
\hline Mould & 0.78 & 10 & 1 & 19 & 401 & 1 \\
\hline Mould & 0.78 & 10 & 1 & 19 & 1148 & 1 \\
\hline Mould & 0.78 & 10 & 1 & 19 & 1012 & 1 \\
\hline Mould & 0.78 & 10 & 1 & 19 & 733 & 1 \\
\hline Mould & 0.78 & 10 & 1 & 19 & 1717 & 1 \\
\hline Mould & 0.78 & 10 & 1 & 19 & 773 & 1 \\
\hline Mould & 0.78 & 10 & 1 & 19 & 445 & 0 \\
\hline Flywheel Housing & 0.81 & 4 & 1 & 20 & 348 & 1 \\
\hline Flywheel Housing & 0.81 & 4 & 1 & 20 & 167 & 1 \\
\hline Flywheel Housing & 0.81 & 4 & 1 & 20 & 1232 & 1 \\
\hline Flywheel Housing & 0.81 & 4 & 1 & 20 & 1118 & 1 \\
\hline Flywheel Housing & 0.81 & 4 & 1 & 20 & 633 & 1 \\
\hline Flywheel Housing & 0.81 & 4 & 1 & 20 & 382 & 1 \\
\hline Flywheel Housing & 0.81 & 4 & 1 & 20 & 321 & 1 \\
\hline Flywheel Housing & 0.81 & 4 & 1 & 20 & 576 & 0 \\
\hline Cylinder & 0.84 & 6 & 0 & 19 & 58 & 1 \\
\hline Cylinder & 0.84 & 6 & 0 & 19 & 37 & 1 \\
\hline Cylinder & 0.84 & 6 & 0 & 19 & 58 & 1 \\
\hline Cylinder & 0.84 & 6 & 0 & 19 & 592 & 1 \\
\hline Cylinder & 0.84 & 6 & 0 & 19 & 1008 & 1 \\
\hline Cylinder & 0.84 & 6 & 0 & 19 & 365 & 1 \\
\hline Cylinder & 0.84 & 6 & 0 & 20 & 1144 & 1 \\
\hline Cylinder & 0.84 & 6 & 0 & 20 & 1430 & 1 \\
\hline Cylinder & 0.84 & 6 & 0 & 20 & 373 & 1 \\
\hline Cylinder & 0.84 & 6 & 0 & 20 & 659 & 1 \\
\hline
\end{tabular}


TABLE 1: Continued.

\begin{tabular}{|c|c|c|c|c|c|c|}
\hline Workpiece Name & Cutting force/KN & $\begin{array}{l}\text { Number of tool } \\
\text { changes } /(N / \mathrm{h})\end{array}$ & Cutting Fluid & Temperature $/{ }^{\circ} \mathrm{C}$ & $\mathrm{TBF} / \mathrm{h}$ & Data type \\
\hline Connect-ing Plate & 1.03 & 14 & 1 & 22 & 234 & 1 \\
\hline Connect-ing Plate & 1.03 & 14 & 1 & 22 & 175 & 1 \\
\hline Connect-ing Plate & 1.03 & 14 & 1 & 22 & 190 & 1 \\
\hline Connect-ing Plate & 1.03 & 14 & 1 & 22 & 151 & 1 \\
\hline Connect-ing Plate & 1.03 & 14 & 1 & 22 & 18 & 1 \\
\hline Connect-ing Plate & 1.03 & 14 & 1 & 22 & 530 & 1 \\
\hline Connect-ing Plate & 1.03 & 14 & 1 & 22 & 349 & 1 \\
\hline Connect-ing Plate & 1.03 & 14 & 1 & 22 & 526 & 1 \\
\hline Connect-ing Plate & 1.03 & 14 & 1 & 22 & 368 & 1 \\
\hline Connect-ing Plate & 1.03 & 14 & 1 & 22 & 174 & 1 \\
\hline
\end{tabular}

TABLE 2: Reliability parameters interval estimations of NC machine tools considering working condition covariate.

\begin{tabular}{lc}
\hline Parameter & Interval estimation \\
\hline$m$ & {$[1.03,1.57]$} \\
$\eta$ & {$[758.72,1797.42]$} \\
$\beta_{1}$ & {$[-0.11,2.45]$} \\
$\beta_{2}$ & {$[-0.00,0.11]$} \\
\hline
\end{tabular}

where $\widehat{\beta}^{* i, B \cdot(\alpha / 2)}$ is the lower limit of interval estimation of the coefficients of the $i$ th working condition covariate at the confidence level $1-\alpha . \widehat{\beta}^{* i, B \cdot(1-\alpha / 2)}$ is the upper limit of interval estimation of the coefficients of the $i$ th working condition covariate at the confidence level $1-\alpha$.

Step 13. According to Step 9, set up confidence level $1-\alpha$ and solve and round $B \cdot(\alpha / 2)$ and $B \cdot(1-\alpha / 2)$, respectively; then the interval estimations of the MTBF of NC machine tools under the $i$ th covariate level are got:

$$
\left[\operatorname{MTBF}^{* i, B \cdot(\alpha / 2)}, \operatorname{MTBF}^{* i, B \cdot(1-\alpha / 2)}\right] \quad i=1,2, \ldots, u,
$$

where $\mathrm{MTBF}^{* i, B \cdot(\alpha / 2)}$ is the lower limit of interval estimation of the MTBF of NC machine tools under the ith covariate level at the confidence level $1-\alpha . \mathrm{MTBF}^{* i, B \cdot(1-\alpha / 2)}$ is the upper limit of interval estimation of the MTBF of NC machine tools under the $i$ th covariate level at the confidence level $1-\alpha$.

\section{Case Study}

The parameter interval estimations are made according to the test data in [1] which is shown in Table 1 for convenience to discuss.

According to Step 1, the working condition covariates' coefficients of the batch of NC machine tools in Table 1 are $\widehat{\beta}_{1}=1.142, \widehat{\beta}_{2}=0.049$, and the parameters of the baseline failure rate function are $m=1.2121, \eta=1156$ when cutting force $F_{c}=0.35 \mathrm{KN}$ and number of tool changes $N_{h}=2$.

The interval estimations of reliability parameters of the batch of NC machine tools are calculated by Steps 2-13. The result is shown in Table 2, where $1-\alpha=97.5 \%$ and $B=1000$.

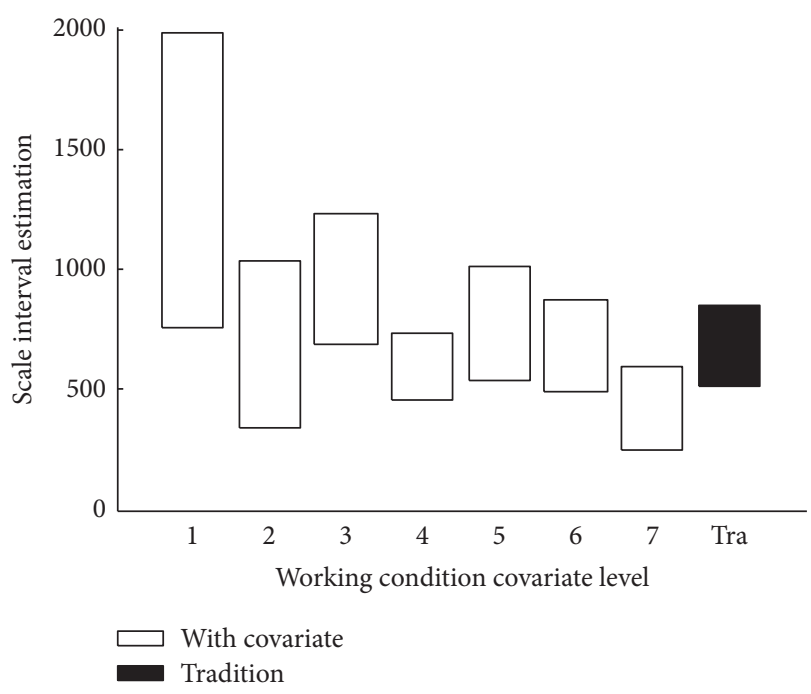

FIGURE 1: Scale parameter interval estimation under each working condition covariate level.

The interval estimation of the scale parameter $\eta$ and MTBF under each working condition covariant level is calculated by Steps 2-13. The result is shown in Table 3.

For clearer, the interval estimations of the scale parameter $\eta$ and MTBF under each working condition covariant level are shown in Figures 1 and 2.

For comparison, model parameters and MTBF interval estimation of NC machine tools obtained by the traditional Bootstrap method, which does not consider the working conditions, are obtained. The detailed procedure of calculation by the tradition method is given in [11], and the corresponding result is shown in Table 4. The interval estimations $m_{\mathrm{Tra}}$ are shown in Figure 1. The interval estimation $\mathrm{MTBF}_{\mathrm{Tra}}$ is shown in Figure 2.

It is seen from Tables 1 and 2 and Figures 1 and 2 that the interval estimation obtained by the traditional method is only one interval estimation, and only under some particular working conditions are they similar to the interval estimation obtained by the new method (e.g., the cutting force $F_{c}=$ $0.84 \mathrm{KN}$ and the number of tool changes $N_{h}=6$ ). There are 
TABLE 3: Reliability parameter interval estimations of NC machine tools under each working condition covariate level.

\begin{tabular}{lcccc}
\hline Covariate level & $F_{c} / \mathrm{KN}$ & $\begin{array}{c}N_{h} \\
\mathrm{n} / \mathrm{h}\end{array}$ & $\eta$ interval estimation & MTBF interval estimation \\
\hline 1 & 0.35 & 2 & {$[758.72,1797.42]$} & {$[713.97,1649.99]$} \\
2 & 0.43 & 17 & {$[342.42,1035.87]$} & {$[320.05,958.97]$} \\
3 & 0.54 & 4 & {$[685.85,1232.43]$} & {$[641.71,1151.08]$} \\
4 & 0.78 & 10 & {$[462.05,733.07]$} & {$[432.67,683.51]$} \\
5 & 0.81 & 4 & {$[532.58,1009.93]$} & {$[493.79,945.30]$} \\
6 & 0.84 & 6 & {$[488.28,872.44]$} & {$[462.02,811.21]$} \\
7 & 1.03 & 14 & {$[251.98,595.01]$} & {$[238.70,558.03]$} \\
\hline
\end{tabular}

TABLE 4: Interval estimation obtained by the tradition Bootstrap method.

\begin{tabular}{lc}
\hline Parameter & Interval estimation \\
\hline$m_{\text {Tra }}$ & {$[0.93,1.47]$} \\
$\eta_{\text {Tra }}$ & {$[514.22,846.03]$} \\
MTBF $_{\text {Tra }}$ & {$[496.76,802.36]$} \\
\hline
\end{tabular}

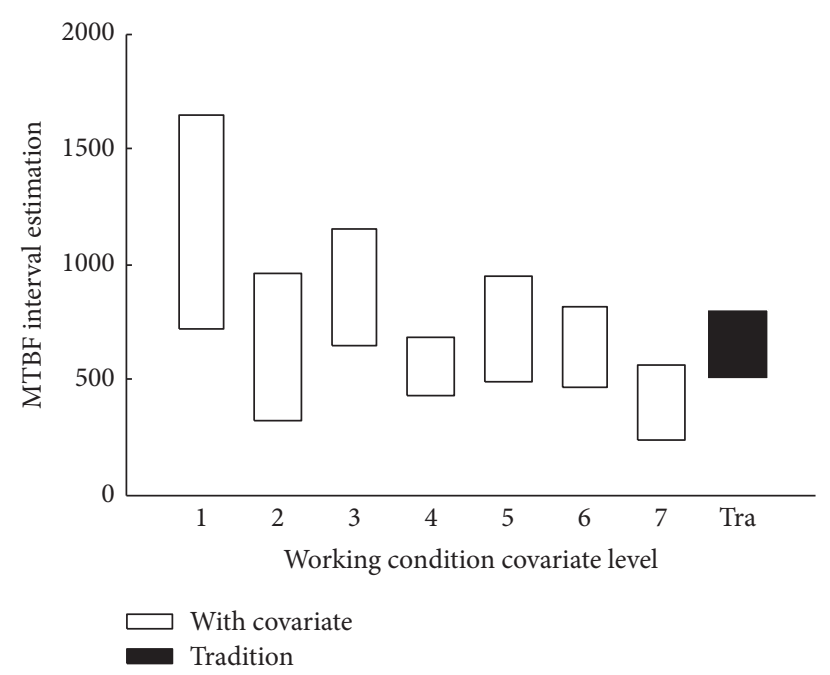

FIGURE 2: MTBF interval estimation under each working condition covariate level.

obvious distinctions in the length and location of the interval estimation of the scale parameter and MTBF under different working condition covariant levels. When $F_{c}=0.35 \mathrm{KN}$ and $N_{h}=2$, the length of the interval estimation of the scale parameter and MTBF is longer and their locations are higher. When $F_{c}=0.78 \mathrm{KN}$ and $N_{h}=10$, the length of the interval estimation of the scale parameter and MTBF is shorter and their locations are lower. It can be concluded from the above facts that the new method can be used to calculate the reliability parameters' interval estimation of NC machine tools considering working condition covariates.

\section{Conclusions}

Given that there may be two or more independent variables (e.g., TBF or working condition covariate) in the reliability model of NC machine tools considering working conditions covariates, this makes other interval estimation methods unfeasible to calculate the interval estimations of the reliability parameters. Considering this problem, the authors propose a new method for the interval estimation of NC machine tools considering working conditions covariates. The resamples are obtained based on the parameters of the test sample collected in the test field. Then the improved Bootstrap method is used to calculate parameter interval estimations of NC machine tools' reliability model. The result of the case study indicated that the new method can be used to calculate reliability parameters' interval estimation of the NC machine tools considering different working condition covariates which cannot be calculated using other methods and provides a more accurate basis for reliability evaluation.

\section{Conflicts of Interest}

The authors declare that they have no conflicts of interest.

\section{Acknowledgments}

Research in this paper was supported by the National Science and Technology Major Project of China (Grant no. 2013ZX04011-012) of reliability promotion of thousands of Chinese CNC machining centers and the Science and Technology Bureau of Jilin City (Grant no. 2013121010).

\section{References}

[1] H. Li, Z. Yang, B. Xu, C. Chen, Y. Kan, and G. Liu, "Reliability evaluation of NC machine tools considering working conditions," Mathematical Problems in Engineering, vol. 2016, Article ID 9842607, 11 pages, 2016.

[2] L. A. Escobar and W. Q. Meeker, "The asymptotic equivalence of the fisher information matrices for type I and type II censored data from location-scale families," Communications in Statistics - Theory and Methods, vol. 30, no. 10, pp. 2211-2225, 2001.

[3] J. G. Yang, Z. M. Wang, G. Wang, and G. Zhang, "Likelihood ratio test interval estimation of reliability indices for numerical control machine tools," Journal of Mechanical Engineering, vol. 48, no. 2, pp. 9-15, 2012.

[4] A. Shafieezadeh and B. R. Ellingwood, "Confidence intervals for reliability indices using likelihood ratio statistics," Structural Safety, vol. 38, pp. 48-55, 2012.

[5] Y. Zhao, J. Yang, and X. B. Ma, Reliability data, analysis [M]., China, Beihang University Press, China, 2009. 
[6] Z. F. Li and W. S. Gao, Mathematical Statistics and Stochastic Processes, Jilin University Press, China, 2000.

[7] A. M. Zoubir and D. R. Iskandler, "Bootstrap methods and applications," IEEE Signal Processing Magazine, vol. 24, no. 4, pp. $10-19,2007$.

[8] H. Z. Li, Reliability Modeling, of NC Machine Tools considering Working Conditions and Testing Period Design, Jilin University, Changchun, China, 2016.

[9] J. L. Simon, Resampling: the new statistics [M]. Resampling stats.

[10] J. F. Lawless, Statistical Models and Methods for Lifetime Data, Wiley-Interscience, 2002.

[11] W. X. Ting, S. Zhou, and J. G. Lin, "Interval estimation comparison between Bootstrap method and classical method," Statistics and Decision, vol. 23, pp. 72-73, 2012. 


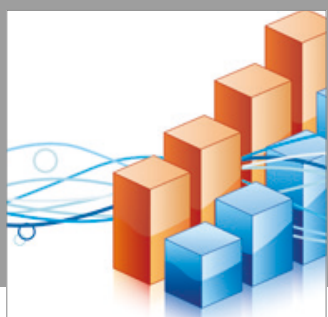

Advances in

Operations Research

vatersals

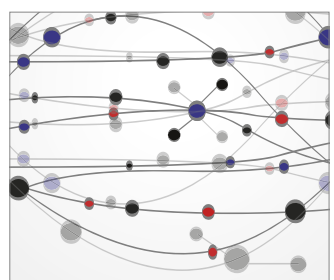

\section{The Scientific} World Journal
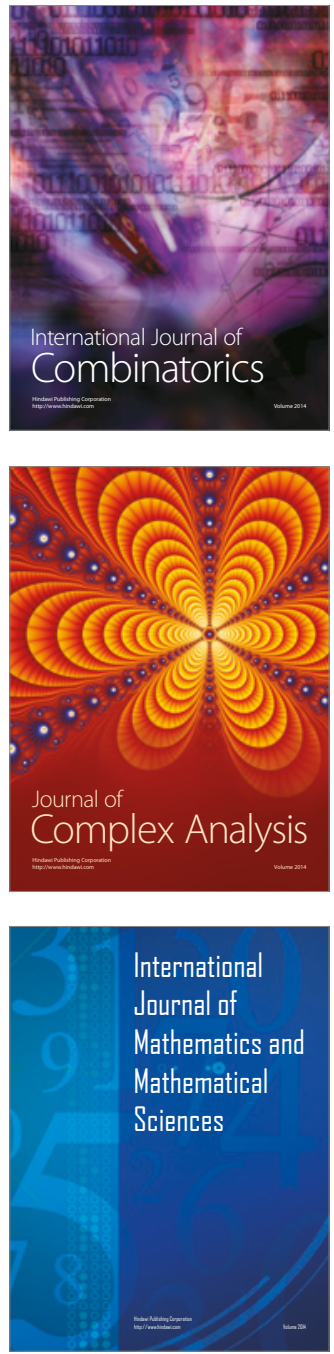
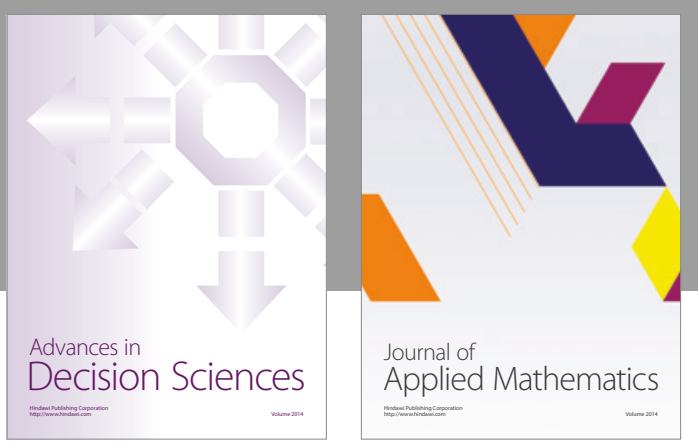

Algebra

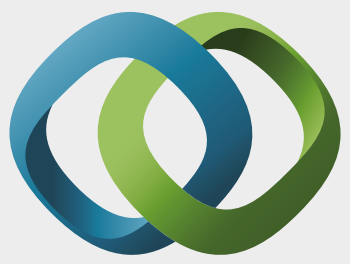

\section{Hindawi}

Submit your manuscripts at

https://www.hindawi.com
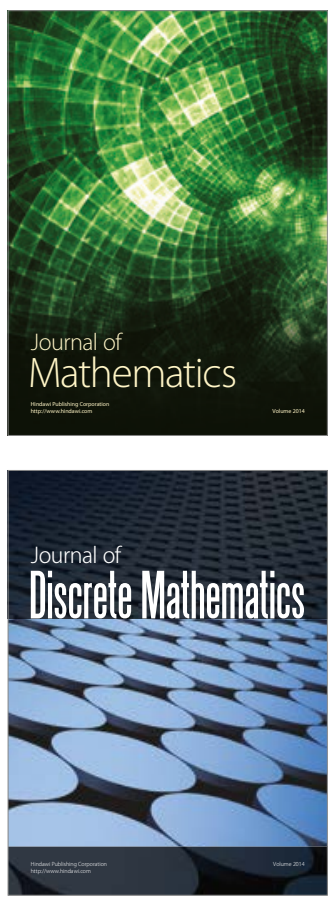

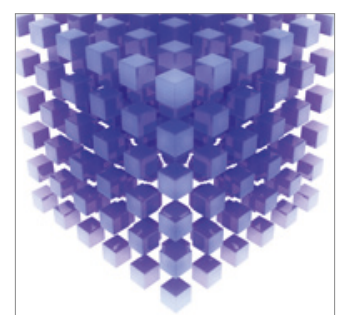

Mathematical Problems in Engineering
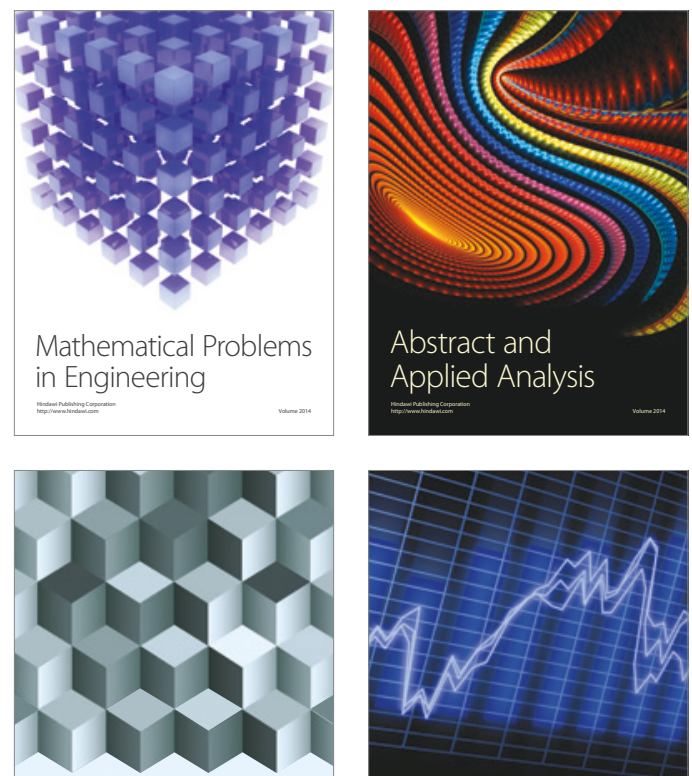

Journal of

Function Spaces

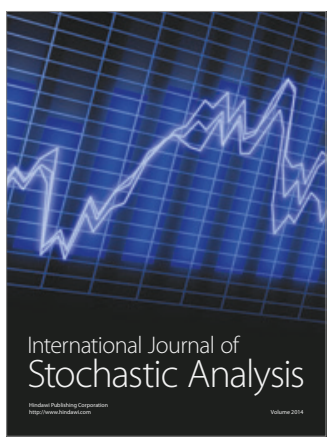

Probability and Statistics
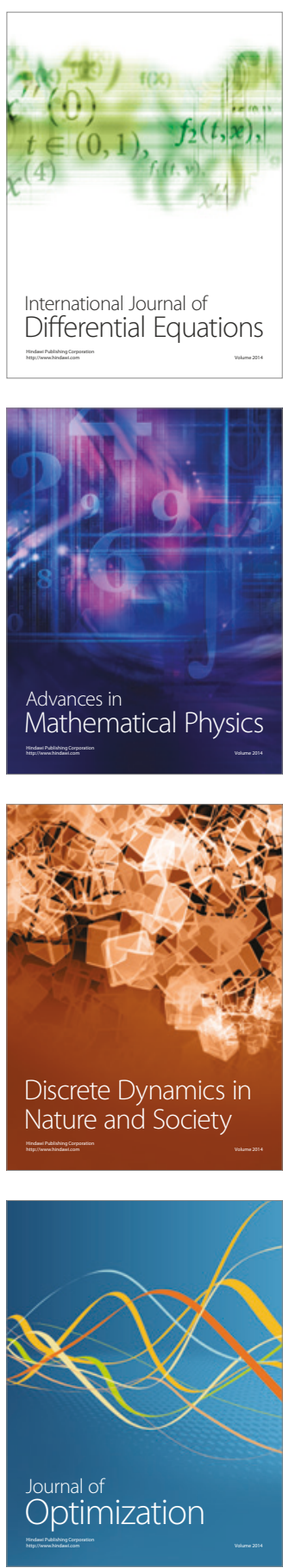